Psychother Psychosom 2016;85:185-186

DOI: $10.1159 / 000443511$

\title{
Feasibility and Outcome of a Brief Cognitive Behaviour Therapy Family Intervention for Patients with Obsessive-Compulsive Disorder: A Pilot Study
}

Karin C.P. Remmerswaal, Neeltje M. Batelaan, Johannes H. Smit, Patricia van Oppen, Anton J.L.M. van Balkom

Department of Psychiatry and $\mathrm{EMGO}^{+}$Institute, VU University Medical Centre, Amsterdam/GGZ inGeest, Amsterdam, The Netherlands

Research has demonstrated the effectiveness of cognitive behaviour therapy (CBT) for patients with obsessive-compulsive disorder (OCD). However, almost half of the treated patients still meet the criteria for OCD at follow-up. A potential strategy for enhancing $\mathrm{CBT}$ is to focus on the family interaction related to obsessive-compulsive (OC) symptoms. Family members commonly respond to OCD in two ways: they accommodate (adapt, participate) and/or antagonize (criticize, oppose). These responses are related to an unfavourable outcome of CBT and to disability in patients and their family members. Hence, focusing treatment on family responses to OCD may enhance therapy outcome and may improve functioning of patients and family members.

Three controlled studies have examined whether family interventions enhance CBT treatment. Results are positive in two studies $[1,2]$ and non-significant in the third [3]. It is remarkable that only one study has focused on antagonistic responses besides accommodative responses [1], showing positive results. However, this study used a group format, and research has indicated superiority of individual family formats [4]. Therefore, we developed a brief CBT family intervention with an individual family format focusing on both antagonistic and accommodative responses. Moreover, contrasting previous studies, we aimed at normalizing the family relationship by encouraging pleasurable, joint activities. This way we positively and contingently reinforced response prevention of OCD.

We aimed to improve: (1) OC symptoms and (2) functioning of patients and family members. The protocol was added to regular CBT and offered to a couple consisting of a patient with OCD and the family member most involved in caring for the patient. This pilot study investigated the feasibility and effectiveness of our brief CBT family intervention.

The protocol involved five sessions of $90 \mathrm{~min}$, planned every other week. Each session included three parts: (1) decreasing an-

\section{KARGER}

E-Mail karger@karger.com www.karger.com/pps

\section{(9) 2016 The Author(s) \\ Published by S. Karger AG, Basel 0033-3190/16/0853-0185\$39.50/0}

This article is licensed under the Creative Commons AttributionNonCommercial-NoDerivatives 4.0 International License (CC BYNC-ND) (http://www.karger.com/Services/OpenAccessLicense). Usage and distribution for commercial purposes as well as any distribution of modified material requires written permission. tagonistic responses of the family members and patients pleading for accommodation by learning effective communication about OCD; (2) response prevention of accommodation by family members, and (3) planning joint activities.

Sixteen patients, 15 partners and 1 mother, were included. The study was conducted at the Academic Outpatient Department for Anxiety Disorders at GGZ inGeest, Amsterdam, the Netherlands.

Inclusion criteria were: (1) OCD of at least moderate severity [Yale Brown Obsessive Compulsive Scale (Y-BOCS) $\geq 16$ ] [5]; (2) accommodation of at least moderate severity [Family Accommodation Scale on the family member (FAS) $\geq 13$ ] [6] and/or antagonism of significant degree [Perceived Criticism Measure on the patient $(\mathrm{PCM}) \geq 4$ ] [7], and (3) medication was allowed but needed to be kept constant during treatment. In addition to the YBOCS, FAS and PCM, pre-tests included assessment of functioning with the World Health Organization Disability Assessment Schedule (WHODAS) [8] and assessment of atmosphere at home with a visual analogue scale ( $0-100$, higher scores indicating a better atmosphere). Post-tests included all mentioned instruments plus assessment of satisfaction with the brief CBT family intervention with a visual analogue scale $(0-100$, higher scores indicating more satisfaction).

Results were examined using two-tailed t tests in the intent-totreat (ITT) sample. Missing values at post-test $(n=5)$ were imputed with pre-test scores. The method of Jacobson and Truax [9] was used to determine reliable change (Y-BOCS decrease $\geq 4$ ) and clinically significant recovery (reliable change and post-test $\mathrm{Y}$ BOCS $\leq 16)$, using data from Frost et al. [10].

Patients had a mean age of 39.4 years (SD 10.8). Ten patients were female $(62.5 \%), 7$ patients had children (43.8\%), 6 patients were employed $(37.5 \%), 14$ patients lived with their family member $(87.5 \%)$ and 7 patients used pharmacotherapy (43.8\%). The mean duration of OCD was 13.9 years (SD 7.1; range $5-31$ years). Patients had received on average 2.8 (range $0-5$ ) previous treatments (psychotherapy and pharmacotherapy). The mean duration of the partner relationship was 14.3 years (SD 13.6; $\mathrm{n}=15$ ). We did not assess psychopathology of family members, which is a limit of this study.

Ten couples completed the family treatment. Six couples dropped out: because their relationship broke up $(n=1)$, because of no show $(\mathrm{n}=2)$ and because of a lack of motivation to change $(n=3)$. Sessions were audiotaped; an independent assessor checked a random sample of $50 \%$ on patient and therapist compliance to the protocol. The therapists offered core interventions according to the protocol; patients adequately conducted most of their homework but were hesitant to practice communication exercises at home.

Table 1 presents the results of the ITT analyses. Results of the ITT and completer analyses were similar. In patients, OC symptoms significantly decreased; 8 patients achieved a reliable change. Of these, 6 recovered. The results of the remaining patients did not 
Table 1. Family treatment results for patients with OCD and family members, ITT sample

\begin{tabular}{|c|c|c|c|}
\hline & $\begin{array}{l}\text { Pre-test } \\
(\mathrm{n}=16)\end{array}$ & $\begin{array}{l}\text { Post-test } \\
(\mathrm{n}=16)\end{array}$ & $t($ d.f. $) ; p$ \\
\hline \multicolumn{4}{|l|}{ Patients } \\
\hline Y-BOCS total & $23.1(4.5)$ & $18.5(6.7)$ & $t(15)=3.43 ; 0.00$ \\
\hline PCM & $6.8(1.6)$ & $6.9(1.3)$ & $\mathrm{t}(15)=-0.46 ; 0.65$ \\
\hline WHODAS & $29.2(16.5)$ & $27.7(3.4)$ & $t(15)=0.41 ; 0.69$ \\
\hline Atmosphere at home & $72.7(22.2)$ & $71.0(19.5)$ & $\mathrm{t}(15)=0.61 ; 0.55$ \\
\hline Satisfaction with family treatment & NA & $83.1(18.7)$ & \\
\hline \multicolumn{4}{|l|}{ Family members } \\
\hline FAS & $19.1(7.6)$ & $12.5(11.0)$ & $t(15)=4.03 ; 0.00$ \\
\hline WHODAS & $14.9(14.7)$ & $11.6(14.6)$ & $\mathrm{t}(15)=2.03 ; 0.06$ \\
\hline Getting along $^{1}$ & $26.6(20.2)$ & $20.0(20.4)$ & $\mathrm{t}(15)=2.48 ; 0.03$ \\
\hline Atmosphere at home & $62.1(19.6)$ & $71.6(17.4)$ & $\mathrm{t}(15)=-3.41 ; 0.00$ \\
\hline Satisfaction with family treatment & NA & $79.6(15.6)$ & \\
\hline
\end{tabular}

Pre- and post-test values are given as mean (SD). ${ }^{1}$ Remaining WHODAS subscales of patients and family members did not change significantly. NA = Not applicable. Bold values: $\mathrm{p}<0.05$.

change significantly, including antagonism. In family members, accommodation significantly decreased and social functioning (WHODAS subscale Getting Along) and atmosphere at home significantly improved. A non-significant trend of improvement in the overall level of functioning appeared.

Both patients and family members were satisfied with the family intervention. They appreciated getting advice about dealing with OCD in the context of their relationship. Both benefited but in different respects. Patients, most of whom had received several previous treatments, experienced a clinically significant reduction of OCD. Family members reduced accommodation, whereby they experienced that the feared consequences did not occur, possibly due to the coping strategies provided. Stopping with antagonism appeared to be difficult; this implied more emotional contact which appeared to trigger threat. Therefore, patients and family members were hesitant to practice communication exercises at home even though they considered them to be helpful. It is possible that five sessions were not sufficient to change antagonistic behaviour patterns, i.e. more sessions are required. The high dropout rate might be explained by the impression of therapists that the brief CBT family intervention got to the root cause of OCD and therefore was hard. More effort is required to inform patients and their family members beforehand about the implications of therapy and to support and motivate them during treatment.

The results of our study indicated a benefit for both patients and family members. Information provided beforehand should address the implications of therapy. Additionally, the protocol should be expanded to elicit antagonistic behaviour change and for motivation and support. Our conclusion is that our treatment package including the brief CBT family intervention is promising and warrants further examination using a controlled design.

Acknowledgements

This study was partly funded by the Netherlands Foundation for Mental Health.

\section{References}

1 Grunes MS, Neziroglu F, Mckay D: Family involvement in the behavioral treatment of obsessive-compulsive disorder: a preliminary investigation. Behav Ther 2001;32:803-820.

$\checkmark 2$ Thompson-Hollands J, Abramovitch A, Tompson MC, Barlow DH: A randomized clinical trial of a brief family intervention to reduce accommodation in obsessive-compulsive disorder: a preliminary study. Behav Ther 2015;46:218-229.

3 Van Noppen B, Steketee G, McCorkle BH, Pato M: Group and multifamily behavioral treatment for obsessive compulsive disorder: a pilot study. J Anxiety Disord 1997;11:431-446.

-4 Thompson-Hollands J, Edson A, Tompson MC, Comer JS: Family involvement in the psychological treatment of obsessive-compulsive disorder: a meta-analysis. J Fam Psychol 2014;28:287-298.

5 Goodman WK, Price LH, Rasmussen SA, Mazure C, Fleischmann RL, Hill CL, Heninger GR, Charney DS: The Yale-Brown Obsessive Compulsive Scale 1. Development, use, and reliability. Arch Gen Psychiatry 1989; 46:1006-1011.

-6 Calvocoressi L, Mazure CM, Kasl SV, Skolnick J, Fisk D, Vegso SJ, Van Noppen BL, Price LH: Family accommodation of obsessive-compulsive symptoms: instrument development and assessment of family behavior. J Nerv Ment Dis 1999;187:636-642.

7 Hooley JM, Teasdale JD: Predictors of relapse in unipolar depressives: expressed emotion, marital distress, and perceived criticism. J Abnorm Psychol 1989;98:229-235.

8 Chwastiak LA, Von Korff M: Disability in depression and back pain evaluation of the World Health Organization Disability Assessment Schedule (WHO DAS II) in a primary care setting. J Clin Epidemiol 2003; 56:507-514.

9 Jacobson NS, Truax P: Clinical significance: a statistical approach to defining meaningful change in psychotherapy research. J Consult Clin Psychol 1991;59:12-19.

10 Frost RO, Steketee G, Krause MS, Trepanier KL: The relationship of the Yale-Brown Obsessive Compulsive Scale (YBOCS) to other measures of obsessive compulsive symptoms in a nonclinical population. J Pers Assess 1995;65:158-168. 